\title{
On Analysis of Tangshan Pre-Hospital Service Efficiency Based on Improved Fuzzy-DEA
}

\author{
Qiaozhi Sang1, Fengzhu Yao², Shuo Hao', Zhennan Zhang1 \\ ${ }^{1}$ Yisheng College, North China University of Science and Technology, Tangshan, China \\ ${ }^{2}$ College of Architectural Engineering, North China University of Science and Technology, Tangshan, China \\ Email: 892965571@qq.com
}

How to cite this paper: Sang, Q.Z., Yao, F.Z., Hao, S. and Zhang, Z.N. (2018) On Analysis of Tangshan Pre-Hospital Service Efficiency Based on Improved Fuzzy-DEA. Open Access Library Journal, 5: e4720. https://doi.org/10.4236/oalib.1104720

Received: June 13, 2018

Accepted: June 26, 2018

Published: June 29, 2018

Copyright () 2018 by authors and Open Access Library Inc.

This work is licensed under the Creative

Commons Attribution International

License (CC BY 4.0).

http://creativecommons.org/licenses/by/4.0/

\begin{abstract}
An allocation model based on improved Fuzzy-DEA is proposed for Tangshan medical health resource. The membership function is particularly introduced to characterize the non-strict partial order relation of the fuzzy numbers, and the internal form of the unit efficiency values is converted into point values. The variance analysis index system $\eta^{2}$ is then used to find factors leading to non-DEA effectiveness and the allocation plan is recommended accordingly: the relevant departments should coordinate the inter-regional resources and optimize the medical staffing level.
\end{abstract}

\section{Subject Areas \\ Fuzzy Mathematics}

\section{Keywords}

Improved Fuzzy-DEA, Indicator Fuzzification, Health Resource Allocation

\section{Introduction}

The centralized pre-hospital command mode is still adopted by 120 emergency centers in Tangshan at present, following the principle of "patient willingness, immediate emergency, and service ability", and uniformly dispatches and directs medical emergency services. The demand for pre-hospital emergency care has gradually expanded with the improvement of the economic level. The lack of first-aid sites, unreasonable site selection, and the fact that the emergency radius cannot effectively cover the demand points, and the problem of first-aid in the far city has been particularly highlighted. In addition, the lack of professional input and inefficient first aid further hindered the development of pre-hospital emergency medical services [1]. Therefore, rational allocation of medical equip- 
ment resources and pre-hospital emergency care systems based on the differences in field conditions still need to be further improved.

DEA (Data Envelopment Analysis) measures the relative efficiency of decision units through linear programming and has been widely used in the analysis of the relative efficiency of health services. Nunamaker first applied DEA to evaluate the efficiency of health resource allocation in 17 hospitals; (Nunamaker T R. Measuring routine nursing service efficiency [J]. Health Service Research, 1983, 18(1): 83-208). Rona proposed that the factor restricting the efficiency of pre-hospital emergency medical services is that the internal structure of supply is misalignment of supply structure rather than insufficient supply on the surface (analysis of the status quo and existing problems of Nanning emergency medical service supply from the perspective of public products); Wan Haohua proposed the evaluation of the nature of standby points around the emergency center in Nanchang. Strengthen the weak areas and improve the service capacity of on-call service points [2]; Zhang Luru et al. using the DEA method based on Pareto optimal principle to calculate relatively low efficiency areas from the perspective of medical resource allocation (the efficiency of medical resource allocation in hospitals in the region Research); Wang Wei and others applied the DEA method to evaluate the service efficiency of 40 hospitals in Huaiyin District and Jingxian County in Jiangsu Province [3]. However, in the emergency scene, the fuzziness index of doping noise is difficult to obtain the exact value of the information. DEA is sensitive to data, and its assessment results are easily affected by inaccurate information such as incomplete information and measurement errors etc.

For the solution of fuzzy DEA, Guo et al. proposed a multiplier model based on fuzzy number comparison rules, converting fuzzy constraints into definite constraints; Leon et al. established the envelope model of the same solution idea; Wu Haiping 14, Zeng Xiangyun et al. In the L_R interval DEA model established by $\mathrm{Wu}$ Wenjiang et al., most models based on comparison rules require that the number of intervals follow a strict partial order relationship, and equations can be equivalent to clear numbers. However, the actual interval fuzzy number is often obtained based on empirical knowledge, and the upper and lower limits of the index cannot be obtained. Based on this, this paper converts the precise output elements into fuzzy numbers, and introduces the membership function to represent its non-strict partial order relationship. By introducing the telescopic coefficient, the membership function was selected to quantitatively characterize the degree of constraint relaxation, and the upper and lower limits of unit efficiency and the best point value were obtained. The model was used to evaluate the efficiency of pre-hospital emergency resource allocation in 16 hospitals in Tangshan City.

\section{Material and Methods}

\subsection{Data Sources}

This article collects information on the number of hospitalized ambulances, 
medical personnel, drivers, and the total amount of medical treatment for 16 pre-hospital service units in Tangshan City according to the hospital's medical record. The data spans from March 1, 2014 to March 2017. April 7. Considering the generality and simplicity of the indicators, and avoiding the mixing of economic and quantitative indicators [4], the hospital is regarded as a decision-making unit for transforming input and output, and the number of vehicles, medical staff, and drivers are used as inputs for the decision unit. The total amount of medical treatment is output. The output indicators, the total diagnosis and treatment fuzzification, introduced the Fuzzy-DEA into the pre-hospital emergency resource allocation efficiency analysis process, and based on this, the Tangshan medical resource allocation plan was proposed.

\subsection{Data Cleanup}

The basic situation of the visiting units is shown in Table 1.

Considering that the input-output index has ambiguity due to its own nature, external subjective cognition, and incomplete information, it limits the accuracy of the sample. The indicators are represented by fuzzy numbers so that the evaluation model is transformed into a fuzzy DEA model.

The output indicators are obscured in this paper, $\alpha$-level cur set of fuzzy number $\tilde{A}$ is noted as $A_{\alpha}=\left\{x \in R \mid \mu_{\tilde{A}}(x) \geq \alpha\right\} \quad\left(A_{\alpha}=\left[A_{\alpha}^{L}, A_{\alpha}^{R}\right]\right)$. Among them, Confidence level $\alpha \in[0,1], A_{\alpha}^{L}$ and $A_{\alpha}^{R}$ denote the upper and lower

Table1. The basic situation of the clinic.

\begin{tabular}{|c|c|c|c|c|c|c|c|c|}
\hline index & $\begin{array}{c}120 \\
\text { Call Center }\end{array}$ & $\begin{array}{c}\text { The eighth } \\
\text { hospital }\end{array}$ & $\begin{array}{l}\text { Second } \\
\text { Hospital }\end{array}$ & $\begin{array}{c}\text { Women and } \\
\text { children } \\
\text { hospital }\end{array}$ & $\begin{array}{l}\text { worker } \\
\text { hospital }\end{array}$ & $\begin{array}{c}\text { North China } \\
\text { University of } \\
\text { Technology Affiliated } \\
\text { Hospital }\end{array}$ & $\begin{array}{c}\text { Kai Luan } \\
\text { hospital }\end{array}$ & $\begin{array}{l}\text { Kai ping } \\
\text { hospital }\end{array}$ \\
\hline \multicolumn{9}{|l|}{ Input indicators } \\
\hline Number of cars & 1 & 1 & 2 & 1 & 6 & 2 & 4 & 2 \\
\hline Number of doctors & 2 & 9 & 8 & 25 & 10 & 6 & 14 & 36 \\
\hline Number of nurses & 1 & 6 & 5 & 5 & 11 & 5 & 14 & 7 \\
\hline Number of drivers & 2 & 4 & 4 & 5 & 12 & 8 & 10 & 5 \\
\hline \multicolumn{9}{|l|}{ Output indicators } \\
\hline \multirow[t]{2}{*}{ General treatment } & 9 & 8109 & 3821 & 2045 & 7942 & 10207 & 4405 & 7227 \\
\hline & $\begin{array}{l}\text { Li Kang } \\
\text { hospital }\end{array}$ & $\begin{array}{l}\text { Ren Min } \\
\text { hospital }\end{array}$ & $\begin{array}{c}\text { Tang Gang } \\
\text { hospital }\end{array}$ & $\begin{array}{l}\text { Railway } \\
\text { hospital }\end{array}$ & $\begin{array}{c}\text { Xie } \mathrm{He} \\
\text { hospital }\end{array}$ & $\begin{array}{l}\text { Zhong Yi } \\
\text { hospital }\end{array}$ & $\begin{array}{c}\text { ninth } \\
\text { hospital }\end{array}$ & $\begin{array}{l}\text { Er wuwu } \\
\text { hospital }\end{array}$ \\
\hline Number of cars & 1 & 4 & 1 & 1 & 2 & 1 & 2 & 2 \\
\hline Number of doctors & 1 & 35 & 4 & 4 & 3 & 10 & 21 & 18 \\
\hline Number of nurses & 1 & 10 & 4 & 6 & 4 & 7 & 20 & 22 \\
\hline Number of drivers & 1 & 7 & 4 & 4 & 3 & 5 & 16 & 13 \\
\hline \multicolumn{9}{|l|}{ Output indicators } \\
\hline General treatment & 1099 & 11,073 & 7764 & 7963 & 9616 & 1017 & 3009 & 2901 \\
\hline
\end{tabular}


boundaries of the internal. $n_{1}$ and $n_{3}$ represent the boundary of observation data floating up and down 10\%, $N_{\alpha}^{L}=n_{1}+\left(n_{2}-n_{1}\right) \alpha, N_{\alpha}^{R}=n_{3}-\left(n_{3}-n_{2}\right) \alpha$. $\tilde{X}_{j}=\left(\tilde{x}_{1 j}, \cdots, \tilde{x}_{m j}\right)^{\mathrm{T}}$ and $\tilde{Y}_{j}=\left(\tilde{y}_{1 j}, \cdots, \tilde{y}_{l j}\right)^{\mathrm{T}}$ indicate the input and output indexes of the decision unit $D M U_{j}(j=1,2, \cdots, n)$.

\section{Research Methods}

DEA [5] is a non-parametric technique that compares production unit analysis efficiencies, especially in the face of multiple inputs and multiple outputs. The selected index is an exact number. However, in reality, we often cannot obtain accurate samples, and we need to select the fuzzy range based on subjective experience. The fuzzy number refers to the regular fuzzy set on the convex set of real numbers $R, \mathrm{~L}-\mathrm{R}$ type fuzzy number requires that:

$$
-\tilde{A}(x)=\left\{\begin{array}{lc}
L\left((a-x) / T_{1}\right) & x \in[\underline{a}, a] \\
1 & x=a \\
R\left((x-a) / T_{2}\right) & x \in[a, \bar{a}] \\
0 & \text { other }
\end{array}\right.
$$

Among them, $L(x)$ and $R(x)$ are strictly monotonically decreasing function of the left and right branches respectively, the fuzzy number can be written as $\tilde{A}=(\underline{a}, a, \bar{a})$, $a$ Is the median or kernel of the fuzzy number $\tilde{A}, \underline{a}$ and $\bar{a}$ being its upper and lower limits. $T_{1}=a-\underline{a}$ and $T_{2}=\bar{a}-a$ are called left and right shape. When the scale is 0 , the fuzzy number degenerates to an exact number, that is, the exact number is a special fuzzy number whose left and right expansion values are 0. The geometric image is shown in Figure 1.

The traditional fuzzy DEA solution method is to transform it into a deterministic planning equation [6], requiring the inequality of two fuzzy numbers to be strictly " $\leq$ " and " $\geq$ ", the upper and lower limits and the intermediate value of the fuzzy number $\tilde{A}$ must be less than (greater than) that in the other number, any fuzzy relationships not allowed. This paper relaxes this constraint by introducing scaling factors $d_{i}$, extending the scope of application of fuzzy DEA.

DEA draws the weights of inputs and outputs based on the sample data itself. The CCR model, originally founded by Charnes, Cooper, and Rhodes, assumes

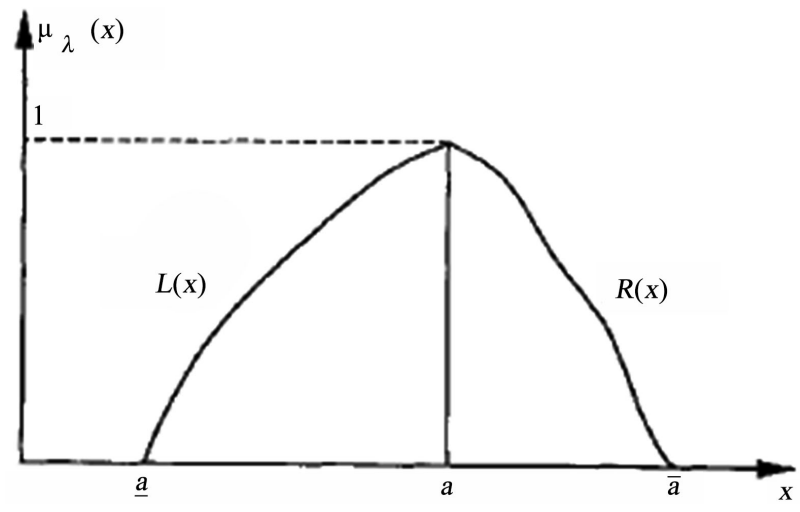

Figure 1. Traditional L-R type fuzzy number. 
that each production unit is always in the stage of constant returns to scale, but this assumption is not true in actual production. The impact of scale was ruled out based on the variable-scale BCC model. Based on the assumptions of minimumness, invalidity, and convexity, when the unit indicator is an L-R interval number, there are production possible sets:

$$
T=\left\{(\tilde{X}, \tilde{Y}) \mid \tilde{X} \geq \sum_{j=1}^{n} \lambda_{j} \tilde{X}_{j}, \tilde{Y} \leq \sum_{j=1}^{n} \lambda_{j} \tilde{Y}_{j}, \Delta \sum_{j=1}^{n} \lambda_{j}=\Delta, \forall \lambda_{j} \geq 0, j=1,2, \cdots, n, \Delta=0 \text { or } 1\right\}
$$

Under the premise of output assumptions, this paper will reduce the input of medical resources and optimize the allocation of primary medical resources as the main way to improve the efficiency of medical services. The input-oriented BBC model is selected:

$$
\begin{gathered}
\min \theta \\
\text { s.t. }\left\{\begin{array}{l}
\sum_{j=1}^{n} \lambda_{j} \tilde{x}_{j} \leq \theta \tilde{x}_{0} \\
\sum_{j=1}^{n} \lambda_{j} \tilde{y}_{j} \geq \tilde{y}_{r k} \\
\lambda_{j} \geq 0, j=1, \cdots, n \\
s^{-} \geq 0 ; s^{+} \geq 0 ;
\end{array}\right.
\end{gathered}
$$

\subsection{Introduction of Flexibility Coefficient and Membership} Function

According to the partial order relation theorem of L-R fuzzy number [7], $A=[\underline{a}, \bar{a}]_{L-R(a)}, B=[\underline{b}, \bar{b}]_{L-R(b)}, A \leq B$ is established if and only if $\underline{a} \leq \underline{b}$, $a \leq b, \bar{a} \leq \bar{b}$. The constraints in the equation are strict "+" and “-” without allowing any violation. We here let the fuzzy indicator take values in different ranges, that is, allow " $\tilde{\leq}$ ” and " $\tilde{\geq}$ ”. A fuzzy set is used to represent the fuzzy relationship $\sum_{j=1}^{n} \lambda_{j} \tilde{x}_{j} \tilde{\leq} \theta \tilde{x}_{0}$. Considering that the membership is 1 when $\tilde{x}_{i}$ takes its central value, that is, $D_{i}(x)=1$. when the fuzzy constraint upper (lower) limit is $\sum_{j=1}^{n} \lambda_{j} \tilde{x}_{j} \leq \theta \tilde{x}_{0}+d_{i}$, the constraint $\sum_{j=1}^{n} \lambda_{j} \tilde{x}_{j} \tilde{\leq} \theta \tilde{x}_{0}$ is not accepted, $D_{i}(x)=0 ; D_{i}(x)$ decrements from 1 to 0 in interval $x \in\left[x_{i}, x_{i}+d_{i}\right]$, indicating that the degree of constraint is gradually relaxed, then The membership function $D_{i}(x)$ is:

$$
D_{i}(x)= \begin{cases}1 & \sum_{j=1}^{n} \lambda_{j} \tilde{x}_{j} \leq \theta \tilde{x}_{0} \\ 1-\left(\sum_{j=1}^{n} \lambda_{j} \tilde{x}_{j}-\theta \tilde{x}_{0}\right) / d_{i} & \theta \tilde{x}_{0} \leq \sum_{j=1}^{n} \lambda_{j} \tilde{x}_{j} \leq \theta \tilde{x}_{0}+d_{i} \\ 0 & \sum_{j=1}^{n} \lambda_{j} \tilde{x}_{j} \geq \theta \tilde{x}_{0}+d_{i}\end{cases}
$$

Among them, $d_{i}\left(d_{i} \in \mathbb{R}, i=1,2, \cdots, n\right)$ is the relaxation coefficient of the 
partial order relation of fuzzy numbers. By introducing this constant index, the strict partial order relationship between fuzzy numbers is relaxed in the form of fuzzy constraints. Let $D=D_{1} \cap D_{2} \cap \cdots \cap D_{n}$, so that all fuzzy numbers are relaxed.

\subsection{The Expression of the Best Decision Linear Programming}

If $\theta_{0}$ is the optimal solution when the fuzzy indicator degenerates into a clear number, the degree of relaxation is $D_{i}(x)=1$. If the degree of membership of the fuzzy constraint $D(x)$ is properly reduced, the constraint condition will relax accordingly, and the target value can be further optimized, which means that the value $\theta$ of the objective function can be reduced. When the constraint relaxes to the widest, $\theta_{1}$ is the optimal value of the fuzzy index when it can accept the constraint edge value, and the fuzzy constraint condition $D(x)$ also approaches 0 accordingly, so the target point value extends to the elastic variation range $\min \left\{\theta_{0}, \theta_{1}\right\} \leq \theta \leq \max \left\{\theta_{0}, \theta_{1}\right\}$, and the target set belongs to The function can be expressed as:

$$
G(x)= \begin{cases}0 & \theta \leq \max \left\{\theta_{0}, \theta_{1}\right\} \\ \left(\theta-\min \left\{\theta_{0}, \theta_{1}\right\}\right) / d_{0} & \min \left\{\theta_{0}, \theta_{1}\right\} \leq \theta \leq \max \left\{\theta_{0}, \theta_{1}\right\} \\ 1 & \theta \geq \min \left\{\theta_{0}, \theta_{1}\right\}\end{cases}
$$

Among them, $d_{0}=\max \left\{\theta_{0}, \theta_{1}\right\}-\min \left\{\theta_{0}, \theta_{1}\right\}$, let fuzzy decision sets be $D_{F}=D \cap G$, The best decision can be expressed as:

$$
\max _{x \in X}(D(x) \Lambda G(x))=\max \{\lambda \mid D(x) \geq \lambda, G(x) \geq \lambda, \lambda \in[0,1]\}
$$

The interval values of the efficiency of the decision unit can then be converted into point values by solving the following equation:

$$
\begin{aligned}
& \max \psi \\
& \left\{\begin{array}{l}
1-\left(\sum_{j=1}^{n} \lambda_{j} \tilde{x}_{j}-\theta \tilde{x}_{0}\right) / d_{i} \geq \psi \quad j=1,2, \cdots, n \\
\left(\theta-\min \left\{\theta_{0}, \theta_{1}\right\}\right) / d_{0} \geq \psi \\
\psi \geq 0 \quad x_{j} \geq 0 \quad \theta \geq 0 \\
\theta_{i}=\psi d_{0}+\min \left\{\theta_{0}, \theta_{1}\right\} .
\end{array}\right.
\end{aligned}
$$

Definition The model's evaluation of efficiency is:

When $\theta_{i}=1.0$, the decision unit $D M U_{i}$ is relatively effective;

When $\theta_{i}<1.0, D M U_{i}$ is relatively ineffective.

\section{Result}

\subsection{Data Envelope Analysis [8]}

Applying the model proposed in the previous section and selected indicators, the efficiency of pre-hospital emergency resource allocation in 16 hospitals in Tangshan area was evaluated. The conventional method of taking cut-offs and the CCR model based on the non-strict partial ordering of fuzzy numbers were 
applied to their efficiency values. Solve. For the cut-off solution method, different confidence level values $\alpha$ are selected, and finally the fuzzy effective ranges $\left[\left(\theta^{*}\right)_{\alpha}^{L},\left(\theta^{*}\right)_{\alpha}^{R}\right]$ and optimal values $\theta$ of the Tangshan City Hospital are respectively obtained ( $\theta$ is the efficiency values obtained by the method in this paper).

Thus, the effective range and optimal effectiveness of the 16 hospitals are shown in Table 2.

The ranking of medical resource allocation efficiency in 16 hospitals was: [120 Call Center, Maternal and Child Hospital, Workers Hospital Affiliated Hospital of North China University of Science and Technology, Kaiping Hospital, Likang Hospital, Ren min Hospital, Railway Hospital, Ninth Hospital, No. 255 Hospital (Parallel)], Eighth Hospital , Second Hospital, [Kai Luan Hospital, Tangshan Hospital (Parallel)], Chinese Medicine Hospital.

\subsubsection{Result Analysis}

As can be seen from Table 2, there are 11 hospitals in Tangshan with a configuration efficiency of 1 , and the DEA effective hospitals are generally higher than the non-DEA effective hospitals in the total number of diagnosis and treatment. The overall efficiency of the hospital in the existing configuration is relatively high. Although the resources of Kai Luan Hospital, TCM Hospital, and effective hospitals are equivalent to or even greater than those of some effective hospitals, the total number of patients under treatment is low, which indicates that the internal medical resources are not fully utilized. There are redundant resources, and improving the quality of technicians at this time has become a key improvement. Part of the lack of resources constrains the increase in output, and technicians are missing and unable to meet the needs of medical staff in a timely manner.

The utilization efficiency of hospital resources in the center of the urban area is generally higher than that of other hospitals. The reason is that high-level technicians and advanced equipment are focused on the urban areas. The infrastructure of the medical institutions in townships and townships is primitive, and the average technicians are far below. Urban area. The medical arrangements in each region are divided and there is a hierarchical relationship.

Special hospitals such as the second hospital are inefficient, because their professional service functions limit the type of disease they see. In urban areas, the prevalence of chronic non-communicable diseases among the residents of the urban areas has led to a drastic reduction in the population served by the urban residents. The fuzzy DEA visually shows the resources and efficiency of each medical institution, and the relevant personnel can control the entry point for improving efficiency.

Comparing the two methods, the traditional method of interception defines the upper and lower limits of the efficiency of the decision-making unit. The relative efficiency of the method to solve this method is higher than the traditional method but the deviation is not large, and effectively compensates for the requirement of the fuzzy number, possessing a strict partial order relationship. 
Table 2. The effective range and optimal effectiveness.

\begin{tabular}{|c|c|c|c|c|c|c|}
\hline$\alpha$ & 0.2 & 0.4 & 0.6 & 0.8 & 1 & $\theta$ \\
\hline$D M U_{1}$ & {$[1,1]$} & {$[1,1]$} & {$[1,1]$} & {$[1,1]$} & {$[1,1]$} & 1 \\
\hline$D M U_{2}$ & {$[9.19 \mathrm{e}-3,1.08 \mathrm{e}-2]$} & {$[4.71 \mathrm{e}-3,5.33 \mathrm{e}-3]$} & {$[3.21 \mathrm{e}-3,3.49 \mathrm{e}-3]$} & {$[2.46 \mathrm{e}-3,2.57 \mathrm{e}-3]$} & {$[2.00 \mathrm{e}-3,2.01 \mathrm{e}-3]$} & 0.00334 \\
\hline$D M U_{3}$ & {$[5.31 \mathrm{e}-3,6.26 \mathrm{e}-3]$} & {$[2.72 \mathrm{e}-3,3.08 \mathrm{e}-3]$} & {$[1.86 \mathrm{e}-3,2.01 \mathrm{e}-3]$} & {$[1.42 \mathrm{e}-3,1.48 \mathrm{e}-3]$} & {$[1.16 \mathrm{e}-3,1.16 \mathrm{e}-3]$} & 0.00193 \\
\hline$D M U_{4}$ & {$[1,1]$} & {$[1,1]$} & {$[1,1]$} & {$[1,1]$} & {$[1,1]$} & 1 \\
\hline$D M U_{5}$ & {$[1,1]$} & {$[1,1]$} & {$[1,1]$} & {$[1,1]$} & {$[1,1]$} & 1 \\
\hline$D M U_{6}$ & {$[1,1]$} & {$[1,1]$} & {$[1,1]$} & {$[1,1]$} & {$[1,1]$} & 1 \\
\hline$D M U_{7}$ & {$[7.82 \mathrm{e}-4,1.82 \mathrm{e}-3]$} & {$[4.09 \mathrm{e}-4,8.94 \mathrm{e}-4]$} & {$[2.84 \mathrm{e}-4,5.85 \mathrm{e}-4]$} & {$[2.2 \mathrm{e}-4,4.30 \mathrm{e}-4$} & {$[1.84 \mathrm{e} 4,3.38 \mathrm{e}-4]$} & 0.00043 \\
\hline$D M U_{8}$ & {$[1,1]$} & {$[1,1]$} & {$[1,1]$} & {$[1,1]$} & {$[1,1]$} & 1 \\
\hline$D M U_{9}$ & {$[1,1]$} & {$[1,1]$} & {$[1,1]$} & {$[1,1]$} & {$[1,1]$} & 1 \\
\hline$D M U_{10}$ & {$[1,1]$} & {$[1,1]$} & {$[1,1]$} & {$[1,1]$} & {$[1,1]$} & 1 \\
\hline$D M U_{11}$ & {$[1.22 \mathrm{e}-2,1.44 \mathrm{e}-2]$} & $6.28 \mathrm{e}-3,7.09 \mathrm{e}-3$ & $4.28 \mathrm{e}-3,4.64 \mathrm{e}-3$ & $3.28 \mathrm{e}-3,3.42 \mathrm{e}-3$ & $2.68 \mathrm{e}-3,2.68 \mathrm{e}-3$ & 0.00043 \\
\hline$D M U_{12}$ & {$[1,1]$} & {$[1,1]$} & {$[1,1]$} & {$[1,1]$} & {$[1,1]$} & 1 \\
\hline$D M U_{13}$ & {$[1,1]$} & {$[1,1]$} & {$[1,1]$} & {$[1,1]$} & {$[1,1]$} & 1 \\
\hline$D M U_{14}$ & {$[7.82 \mathrm{e}-4,1.51 \mathrm{e}-3]$} & {$[4.09 \mathrm{e}-4,7.41 \mathrm{e}-4$} & {$[2.84 \mathrm{e}-4,4.85 \mathrm{e}-4]$} & {$[2.22 \mathrm{e}-4,3.56 \mathrm{e}-4]$} & {$[1.84 \mathrm{e}-4,2.80 \mathrm{e}-4]$} & 0.00038 \\
\hline$D M U_{15}$ & {$[1,1]$} & {$[1,1]$} & {$[1,1]$} & {$[1,1]$} & {$[1,1]$} & 1 \\
\hline$D M U_{16}$ & {$[1,1]$} & {$[1,1]$} & {$[1,1]$} & {$[1,1]$} & {$[1,1]$} & 1 \\
\hline
\end{tabular}

\subsubsection{Variance Analysis of Influencing Factors}

According to J.Cohen, an indicator system can be used to assess the effect of variance analysis [9], namely:

$$
\eta^{2}=\frac{S S_{\text {between groups }}}{S S_{\text {overall }}}
$$

That means the proportion of the sum of squares between the groups after the experimental treatment in the sum of squares of the overall population. The larger the value, the greater the effect of the experiment reflected. $\eta^{2}=0.01$ as the smaller effect, $\eta^{2}=0.06$ denotes the medium effect, and $\eta^{2}=0.14$ presents that the effects are significant. The meaning is clear and easy to understand, so it was adopted by SPSS as an index of the size of the relevant experimental effect in the general linear model module (Chinese version shows "partial Eta square"; English version is translated as "Partial Eta Squared"), as can be seen from Table 3. There was no significant difference in the number of vehicles dispatched by non-DEA effective hospitals $\left(\eta^{2}=0.17\right)$, and there was a significant difference in the allocation of medical staff and the number of visits. Despite the introduction of a large number of medical personnel in hospitals in recent years, their service capabilities and professional operating capabilities are still insufficient, which is the main bottleneck leading to low hospital efficiency. Kailuan Hospital and Tangshan Hospital have the same efficiency, but Kailuan Hospital has significantly higher number of drivers and drivers than Tangshan Hospital, which also proves the reliability of the above analysis. The reason is 
Table 3. Inter-subject effect test.

\begin{tabular}{cc}
\hline non-DEA effective unit & Partial Eta Squared \\
\hline Number of cars & 0.170 \\
Number of doctors & 1.000 \\
Number of nurses & 1.000 \\
Number of drivers & 0.388 \\
General treatment & 1.000 \\
\hline
\end{tabular}

that the Eighth Hospital, the Second Hospital, the Kailuan Hospital, the Tangshan Hospital, and the Chinese Medicine Hospital are basically in the same urban traffic environment. The increase in the number of vehicles does not have a significant impact on the patient's transportation. However, the changes in the allocation of medical staff will greatly affect the speed of the treatment of patients and the efficiency of the distribution of medical resources. The total diagnosis and treatment reflect the total amount of patients treated, so the relevant departments should coordinate the allocation of resources between regions, and optimize service levels. This is the best entry point for improving the efficiency of the hospital.

\section{References}

[1] Hou, Z.Y., Meng, Q.Y., Yuan, Y., et al. (2010) Research on the Current Status of Basic Medical Service Provision Capacity in Urban Communities. Chinese Health Economics, 29, 41-43.

[2] Wan, Y.H. (2016) Spatial Settings and Optimization Ideas of Emergency Standby Point in Nanchang Emergency Center. Clinical Emergency Department, 17, 479-481.

[3] Wang, X.X., Zhu, M., Zhang, S.D. and Chen, J.Y. (2014) Evaluation of the Efficiency of Basic Medical Services in Township Hospitals Based on DEA. Chinese Health Economics, 33, 75-77.

[4] Zeng, X.Y. and Wu, Y.H. (2001) L-R Interval DEA Model and Its Transformation. Chinese Journal of Management Engineering, No. 01, 11-13+4-3.

[5] Huang, Y.X. and Hu, Z.L. (2004) Application of Data Envelopment Analysis in Evaluating Input and Output Efficiency of Township Health Centers. Chinese Health Economics, 23, 61-64.

[6] Li, J.G. and Liu, Y.Q. (1996) DEA Model with Triangular Fuzzy Number Elements. Journal of Systems Engineering, 11, No. 4.

[7] Jaroslav, R. and Josed, R. (1985) Inequality Relation between Fuzzy Numbers and Its Use in Fuzzy Optimization. Fuzzy Sets and Systems, 16, 123-138. https://doi.org/10.1016/S0165-0114(85)80013-0

[8] Wei, Q.L. (2012) Data Envelopment Analysis Model for Evaluation of Relative Validity-DEA and Network DEA. China Renmin University Press, Beijing.

[9] Cohen, J. (1988) Statistical Power Analysis for the Behavioral Sciences. Psychology Press, New York. 